\title{
Magel2 gene mutation and its associated phenotypic features in a five-month-old female
}

\begin{abstract}
We present a unique case of a patient with MAGEL2 mutation, her phenotypic features, and clinical course in comparison to Prader Willi Syndrome's phenotype and course. Dysmorphic facial features with esotropia and micrognathia, feeding difficulties with poor suck, neonatal hypotonia, ineffective thermoregulation, sleep disturbance, small hands and feet are the features that were present in our patient at birth and prompted genetic evaluation. The melanoma antigen (MAGE) gene family of ubiquitin ligase regulators contains the MAGEL2 gene located within the Prader Willi locus. Although the MAGEL2 gene is located within the Prader-Willi Syndrome (PWS) locus, it is important to differentiate its associated phenotypic features not typically shared by PWS. At early childhood, those with PWS suffer hyperphagia, with the potential development of life-threatening obesity, diabetes, and right-sided heart failure into adulthood. Given the severity of these outcomes, we aim to describe the unique phenotypic features of MAGEL2 mutation that guide clinical suspicion and early intervention for management of its complex presentation.
\end{abstract}

Keywords: MAGEL2, prader-willi syndrome, phenotypic features, micrognathia, PWS
Volume 8 Issue I - 2018

\author{
Nina Poliak, ${ }^{1,2,3}$ Preethi Rajan ${ }^{2}$ \\ 'Department of Pediatrics, Lehigh Valley Health Network, USA \\ ¿University of South Florida Morsani College of Medicine, USA \\ ${ }^{3}$ Comprehensive Medical Consulting, USA
}

\section{Correspondence: Nina Poliak, MD, MPH, Department of Pediatrics, Comprehensive Medical Consulting, 125 Birch Avenue, Bala Cynwyd, USA, Email nxpoliak@gmail.com}

Received: January 30, 2018 | Published: February 09, 2018
Abbreviations: PWS, prader willi syndrome; MAGE, melanoma antigen; DOL, day of life; GERD, gastroesophageal reflux disease; G, gastrostomy; GJ, gastrojejunostomy

\section{Introduction}

Melanoma antigen L2 (MAGE-L2) belongs to the approximately 60 gene MAGE family, which is divided into two subsets based on chromosomal location and expression pattern. MAGE proteins act as ubiquitin ligase regulators in conjunction with E3 RING ubiquitin ligases to form MAGE-RING ligases (MRLs). MAGEL2 has elevated expression in the hypothalamus and functions within a multi-subunit protein complex, which consists of MAGEL2, the TRIM27 E3 ubiquitin ligase, and the USP7 deubiquitinating enzyme. This complex is critical to the cellular process of recycling membrane proteins from endosomes through the retromer sorting pathway via ubiquitination and activation of the WASH actin nucleation promoting factor. ${ }^{1}$

The physiological functions of MAGEL2 have been studied in several mouse models. Mice deficient in MAGEL2 expression in the suprachiasmatic nucleus show alterations in circadian rhythm and reduced motor activity, which may be mediated by the associated reduction in levels of orexin and orexin-positive neurons. These alterations in circadian rhythm appear linked to decreased fertility and early infertility in both male and female MAGEL-null mice. ${ }^{2}$ MAGEL2null mice also display neonatal growth retardation, significant weight gain status post weaning, and changes in metabolism in adulthood with increased adiposity. ${ }^{3}$ Recently, a patient initially diagnosed with Opitz-C syndrome, a rare heterogeneous disease associated with psychomotor delay, trigonocephaly, and facial dysmorphisms, was identified to have a de novo nonsense mutation in MAGEL2. ${ }^{4}$ In addition, two families with lethal arthrogryposis multiplex congenita were found to have truncating mutations in MAGEL2. ${ }^{5}$ MAGEL2 has been most extensively studied in relation to the neurodevelopmental syndromes such as Prader-Willi Syndrome, and to a lesser extent, Schaaf-Yang Syndrome. ${ }^{6}$ MAGEL2 is located on the proximal long arm of human chromosome 15 (15q11-q13), within the locus for Prader-Willi Syndrome. In Prader Willi Syndrome, MAGEL2R undergoes maternal imprinting and is typically paternally deleted or mutated. Its other two genetic subtypes are maternal uniparental disomy (20-30\% of cases) and imprinting defect (1-3\%). ${ }^{7}$

Elucidating the phenotypic features of candidate genes, such as MAGEL2, will allow for improved targeted intervention. It is imperative that the associated clinical features of MAGEL2 mutation be identified as early as possible that the severity of the associated syndrome are managed appropriately to improve health and long-term outcome for patients with this mutation.

\section{Case presentation}

We present a 5-month-old female who was a product of a late preterm pregnancy and was born vaginally to non-consanguineous parents. The patient's birth weight was 2700 grams. At 4.5 hours of life the patient was transferred to neonatal intensive care unit (NICU) due to apnea, abnormal eye movements, and hypotonia. The patient's face appeared dysmorphic with microcephaly (head circumference of $32.5 \mathrm{~cm}$ ), micrognathia and esotropia. She also appeared to have disproportionately small hands and feet. The patient was lethargic with no interest in feedings and poor suck/gag reflex. She was placed on feeds through a nasogastric tube. Her laboratory workup was significant for hypoglycemia and hypocalcemia. On the first day of life, an MRI showed Grade I intraventricular hemorrhage bilaterally and a hemorrhage within the pituitary gland. Her EEG showed bitemporal sharps and complexes concerning for subclinical seizures.

At Day 10 she was weaned to room air but continued having difficulty swallowing secretions with poor suck/gag reflexes and GERD. By week 4, the patient was less hypotonic but remained on tube feeds due to high aspiration risk without suck/gag reflex. The patient also had poor weight gain, intermittent pulmonary difficulties and was on and off oxygen and CPAP over the course of her hospital stay. Her ineffective thermoregulation was also monitored throughout. An Echocardiogram was done and revealed an atrial septal defect. At DOL 66, the G tube was placed with umbilical hernia repair. Physical therapist worked with the patient throughout hospital stay to assist with generalized hypotonia, head lag, and tightening of hands. Patient also had testing for level of Insulin-like-Growth-Factor-BindingProtein 3, which was low at $520 \mathrm{ng} / \mathrm{ml}$ (Normal: 1039-3169 ng/ml). At 
DOL 75, the patient was discharged home with portable suction and G-tube. Patient was also referred to early intervention services. After discharge, the patient has been followed by pulmonary, endocrine, surgery, neurology, and gastroenterology. The patient's weight at discharge was $3975 \mathrm{~g}$. At 3 months, neurology reported that patient had diffuse hypotonia with poor neck control, continued tightening in the hands with limited mobility, and inability to fix or follow with eyes. Due to concerns for poor gastric emptying and possible aspiration, Interventional Radiology changed the $\mathrm{G}$ tube to a gastrojejunostomy (GJ) tube. With regards to pulmonary concerns, the patient underwent a sleep study, during which she had an episode of apnea lasting 15 seconds. The patient was admitted twice for pneumonia and continues to require daily nebulizer treatments, with nasal oxygen and CPAP as needed.

At 6 days of life, the baby underwent genetic testing for the MAGEL2c1912c pathogenic mutation, based on the history of the same mutation in her three-year-old brother and father. The results came back positive. The patient's mother met with a genetic counselor prenatally at $13 \mathrm{w} 2 \mathrm{~d}$ and was educated that the recurrence risk for the condition was $50 \%$. She declined all prenatal genetic testing, including amniocentesis, cell-free DNA testing, sequential screen, and detailed fetal anatomy scan. The patient's father has the same mutation but is asymptomatic. The patient's two-year-old brother tested negative for the MAGEL2c1912c pQ638 mutation. The patient's three-year-old brother tested positive for the same mutation and has a history of Pierre Robin sequence, hydronephrosis, microphallus, panhypopituitarism, GERD, dysphagia with G-tube and Nissen, asthma, OSA, micrognathia, developmental delay and autism.

\section{Discussion}

This patient's phenotypic presentation is consistent with some of both major and minor criteria of Prader Willi syndrome (Table 1). Consistent with major criteria, our patient had neonatal hypotonia with poor suck, facial dysmorphism, and feeding problems. Consistent with minor criteria, our patient had infantile lethargy, small hands and feet, thick saliva, and sleep disturbance. ${ }^{8}$ However, the patient also had several features outside of the major and minor criteria associated with Prader Willi syndrome, evidencing the unique features associated with the MAGEL2 mutation. Some of these features are also shared by her brother who has the same mutation. For instance, our patient had an MRI with suspicion for pituitary hemorrhage, and concern for developing hypopituitarism was expressed. The patient's brother also has panhypopituitarism. The patient also had a low level of IGFBP-3, indicative of pituitary dysfunction as well. The patient also suffered ineffective thermoregulation, which further complicated her clinical course. The patient had EEG changes, which showed bitemporal sharps and complexes warranting a work up for subclinical seizures. Our patient also had tightening of the hands and feet, which was concerning for potential arthrogryposis. Her brother also has arthrogryposis, a feature which has been cited in one other case of MAGEL2 mutation. ${ }^{6}$ It was noted that she has abnormal eye movements, not characterized as esotropia, which will further complicate her development. The patient's micrognathia, an unique feature associated with MAGEL2, has also been detrimental to her episodic apnea and feeding difficulties. Finally, with regards to cardiac findings, our patient has an atrial septal defect. This feature is not part of the major or minor criteria of PWS, but is consistent with the finding of cardiac anomalies as the most common congenital defect in PWS. ${ }^{9}$ Our case underscores the importance of identification of features of Prader Willi Syndrome and other similar syndromes, in association with early genetic testing and early intervention.
Table I Phenotypic Features Present in Our Patient, Prader Willi Syndrome, and of MAGEL2 Gene Mutation

\begin{tabular}{|c|c|c|c|}
\hline Our Patient's Features & $\begin{array}{l}\text { Prader Willi } \\
\text { Major Criteria }\end{array}$ & $\begin{array}{l}\text { Prader Willi } \\
\text { Minor Criteria }\end{array}$ & Magel2 \\
\hline Facial Dysmorphism & $x$ & & \\
\hline Developmental Delay & $x$ & & \\
\hline Feeding Problems/Failure to & $x$ & & \\
\hline \multicolumn{4}{|l|}{ Thrive in Infancy } \\
\hline Infantile Central Hypotonia & $x$ & & \\
\hline Infantile Lethargy & & $x$ & \\
\hline Abnormal Eye Movements & & X (Esotropia) & $x$ \\
\hline \multicolumn{4}{|l|}{ (Not Well Characterized) } \\
\hline Small Hands and Feet & & $x$ & \\
\hline Sleep & Disturbance/Sleep & $x$ & \\
\hline \multicolumn{4}{|l|}{ Apnea } \\
\hline Thick,Viscous Saliva & & $x$ & \\
\hline ASD & & & $\mathrm{x}$ \\
\hline Ineffective & & & $\mathrm{x}$ \\
\hline \multicolumn{4}{|l|}{ Hypo thermoregulation } \\
\hline Micrognathia & & & $x$ \\
\hline Pituitary & Hemorrhage & & $x$ \\
\hline \multicolumn{4}{|l|}{ Possible Panhypopituitarism } \\
\hline EEG Changes & & & $x$ \\
\hline Tightening of the Hands and & & & $x$ \\
\hline \multicolumn{4}{|l|}{ Feet } \\
\hline (Possible Arthrogryposis) & & & \\
\hline
\end{tabular}

Mutations in MAGEL2 portend lifelong management of associated complications, including global developmental delays, behavioral problems, and the dichotomous nature of nutritional/ growth complexities. In later infancy or early childhood patients with Prader-Willi syndrome, the period of hyperphagia ensues, ${ }^{10}$ with PWS as the most common known cause of childhood morbid obesity without treatment. In adulthood, patients with PWS can suffer behavioral problems, short stature, diabetes, sleep abnormalities including obstructive sleep apnea, and right-sided heart failure, with death typically within the fourth decade of life. Although our patient shares several features of Prader-Willi syndrome, her course through her childhood is incompletely defined, as evidenced by the disparities in presentation between her and her brother and the several discrepant features from Prader Willi diagnostic criteria. It is vital for parents to receive appropriate education regarding prenatal testing as well as anticipatory guidance at each phase of childhood. ${ }^{11}$ It is pivotal for different pediatric specialists to work together to manage syndromes less commonly described in order to improve patients' long-term outcome. Moving forward, it will be critical to continue monitoring our patient's development, to see which of these features she will share and what features will be unique to MAGEL2 mutation, and how to manage her unique presentation appropriately.

\section{Conflicts of interest}

The authors certify that they do not have any affiliation with or financial involvement in any organization or entity with a direct financial interest in the subject matter or materials discussed in the paper (e.g., employment, consultancies, stock ownership, honoraria, and expert testimony). They do not have any commercial or proprietary interest in any drug, device, or equipment mentioned in the paper. They declare that they do not have any competing interests. No financial support was used for this work. No previously published figures or tables were used in this paper. 


\section{Acknowledgments}

None.

\section{Conflicts of interest}

None.

\section{Funding}

None.

\section{References}

1. Lee AK, Potts PR. A Comprehensive Guide to the MAGE Family of Ubiquitin Ligases. J Mol Biol. 2017;429(8):1114-1142.

2. Kozlov SV, Bogenpohl JW, Howell MP, et al. The imprinted gene Magel2 regulates normal circadian output. Nat Genet. 2007;39(10):1266-1272.

3. Bischof JM, Stewart CL, Wevrick R. Inactivation of the mouse Magel2 gene results in growth abnormalities similar to Prader-Willi syndrome. Hum Mol Genet. 2007;16(22):2713-2719.

4. Urreizti R, Cueto Gonzalez AM, Franco Valls H, et al. A De Novo Nonsense Mutation in MAGEL2 in a Patient Initially Diagnosed as Opitz-C: Similarities Between Schaaf-Yang and Opitz-C Syndromes. Sci Rep. 2007;7:44138.
5. Mejlachowicz D, Nolent F, Maluenda J, et al. Truncating Mutations of MAGEL2, a Gene within the Prader-Willi Locus, Are Responsible for Severe Arthrogryposis. Am J Hum Genet. 2015;97(4):616-620.

6. Fountain, Schaaf CP. Prader Willi Syndrome and Schaaf-Yang Syndrome: Neurodevelopmental Diseases Intersecting at the MAGEL2 Gene. Diseases. 2016;4(1):E2.

7. Angulo MA, Butler MG, Cataletto ME. Prader-Willi syndrome: a review of clinical, genetic, and endocrine findings. $J$ Endocrinol Invest. 2015;38(12):1249-1263.

8. Gunay Aygun M, Schwartz S, Heeger S, et al. The changing purpose of Prader-Willi syndrome clinical diagnostic criteria and proposed revised criteria. Pediatrics. 2001;108(5):E92.

9. Torrado M, Foncuberta ME, Perez MF, et al. Change in prevalence of congenital defects in children with Prader-Willi syndrome. Pediatrics. 2013;131(2):e544-e549.

10. McCandless, Shawn E. Health supervision for children with PraderWilli syndrome. Pediatrics. 2011;127(1):195-204.

11. Butler MG. Prader-Willi Syndrome: Obesity due to Genomic Imprinting. Curr Genomics. 2011; 12(3):204-215. 